\title{
Antonin PERBÒsC, 2013, Au país de la gata blanca. Contes populars amassats a Combarogèr
}

Edicion establida per Josiana Bru e Joan Eygun, s.l., Letras d'òc, 250 p., ISBN : 978-2-916718-46-0.

Jean-Pierre Cavaillé

\section{OpenEdition}

\section{Journals}

\section{Édition électronique}

URL : https://journals.openedition.org/clo/1759

DOI : $10.4000 /$ clo. 1759

ISSN : 2266-1816

Éditeur

INALCO

\section{Édition imprimée}

Date de publication : 2 janvier 2012

ISBN : 978-2-85837-212-2

ISSN : 0396-891X

\section{Référence électronique}

Jean-Pierre Cavaillé, « Antonin PERBòsc, 2013, Au país de la gata blanca. Contes populars amassats a

Combarogèr », Cahiers de littérature orale [En ligne], 72 | 2012, mis en ligne le 14 avril 2015, consulté le 02 juillet 2021. URL : http://journals.openedition.org/clo/1759 ; DOI : https://doi.org/10.4000/clo.1759

Ce document a été généré automatiquement le 2 juillet 2021.

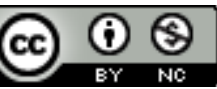

Cahiers de littérature orale est mis à disposition selon les termes de la Licence Creative Commons Attribution - Pas d'Utilisation Commerciale 4.0 International. 


\section{Antonin PERBÒSC, 2013, Au país de la gata blanca. Contes populars amassats a Combarogèr}

Edicion establida per Josiana Bru e Joan Eygun, s.l., Letras d'òc, 250 p., ISBN : 978-2-916718-46-0.

Jean-Pierre Cavaillé

1 Ce recueil de contes est le fruit d'une démarche pédagogique très originale, novatrice et courageuse d'un instituteur hors du commun, dans les années 1899-1905, en poste à l'école de Comberouger dans le Tarn-et-Garonne. Démarche originale, avant-gardiste même, puisqu'elle a consisté à ériger les enfants en enquêteurs ethnographes, en «folkloristes » disait-on alors, collectant tous les éléments de tradition orale dans leur langue natale (gascon mâtiné de languedocien, Comberouger se tenant sur la limite interdialectale). Mais aussi démarche courageuse, quand on sait que la mutation d'Antonin Perbosc dans ce petit village de Lomagne en 1894 était le résultat d'une sanction disciplinaire pour des raisons similaires ; on lui avait en effet reproché d'avoir donné « des devoirs patois » à ses élèves.

2 Les enfants déploient leur activité dans le cadre d'une société scolaire créée ad hoc en 1899, sur le modèle associatif que la loi de 1901 officialisera juste après : la «société traditionniste ». Le principe est simple et de bon sens : " en s'adressant à tous les enfants et en dirigeant convenablement leurs travaux, on peut recueillir en peu de temps toutes les traditions » d'un territoire donné (les citations de Perbosc sont tirées de la préface de l'ouvrage par Josiane Bru et d'un article du même auteur : «Perbosc et l'enquête folklorique $»^{1}$ ). Perbosc tient à préciser: «je ne suis que le directeur de ce travail »; l'enquête, « ce sont mes élèves, une quinzaine de garçons et de filles de 10 à 13 ans qui la font». La direction consiste en la transmission d'une déontologie de l'exactitude: ces contes, proverbes, histoires, «il leur est recommandé de les noter avec la plus grande fidélité et en s'abstenant d'une façon absolue d'y apporter des modifications »; « ces contes ne doivent pas être enjolivés, sous aucun prétexte ». En effet, même «les contes les plus informes peuvent présenter un grand intérêt ». Et, 
alors même que Perbosc est un artisan majeur de la normalisation graphique de l'occitan, il demande aux élèves d'user d'une "graphie purement phonétique »; en effet, "nous ne demandons ici à nos textes que de refléter aussi exactement que possible des traditions orales ».

Perbosc fut le premier étonné par la quantité et la qualité des matériaux rassemblés en peu de temps par cette recherche collective dans laquelle on sait grâce aux souvenirs que, devenus adultes, ils ont laissés à leurs proches, que les enfants se sont impliqués corps et âme. "Les résultats, écrit Perbosc, que j'ai obtenus ont dépassé toutes mes espérances »; " une seule gamine de 11 ans m'a donné quatre contes qui feraient plus de trente pages d'imprimerie, et cela de qualité supérieure ». Il constate d'ailleurs que " ce sont surtout les filles qui font d'excellentes folkloristes".

«Les enfants dont l'esprit est éveillé par l'étude du folklore s'habituent à observer, à réfléchir [...]. Enfin, au point de vue de l'instruction proprement dite, les exercices de composition, de traduction, de comparaison de textes présentent la plus grande utilité pour l'enseignement du français. » Ainsi, Perbosc défend-il le bénéfice pédagogique de l'entreprise en des termes qui auraient pu la rendre acceptable et même auraient dû l'ériger en modèle. Il n'en fut rien. Ces prodromes de ce que l'on appellera beaucoup plus tard la « pédagogie active » demeurèrent largement incompris des contemporains. Malgré tous ses efforts pour diffuser des sociétés traditionnistes enfantines, l'initiative de Perbosc resta en effet sans lendemain. Lui-même ne publia d'ailleurs pas ces contes dans leurs versions originales, brutes peut-on dire, alors qu'il disait lui-même que « les contes bien conservés sont parfaits ». On peut se demander à ce propos si, justement, ce caractère brut, non policé, non enjolivé, qui donne tout son prix à ce recueil, n'en faisait pas un objet inapproprié à la diffusion imprimée dans les années 1900, et cela aux yeux mêmes de son initiateur, dont on peut admirer le travail d'orfèvre sur la langue dans son œuvre propre (voir par exemple notre compte rendu du Libre dels ausels).

Le texte retenu par J. Eygun et J. Bru est donc inédit; il s'agit du manuscrit conservé à la Bibliothèque municipale de Toulouse (ms 1452), un cahier noir aux feuilles quadrillées que l'on peut désormais consulter en ligne, et qui ne contient pas moins de cent quinze textes transcrits en occitan (sur ce point la fiche en ligne de la Bibliothèque, qui porte "Contes populaires, en français, recueillis à Comberouger ", est erronée ${ }^{2}$ ), chacun le plus souvent suivi d'une traduction. Les écritures sont celles de l'instituteur et de l'une de ses élèves, la "présidente» de la Société traditionniste, Marie Tournié (treize ans en 1900). Elle sera couturière sa vie durant à Beaumont-deLomagne. Destin signé, mais pour ces pauvres agriculteurs de Comberouger, une réussite; nous le savons par l'un des contes que Marie a elle-même recueilli dans sa famille, une version du Petit Poucet, qui se termine par ces mots : "Le petit pocet se metec factur, una de sas sòrs se metec costurièra, prenguegan un mestièr, e venguegan plan riches» («Le petit poucet devint facteur, l'une de ses sœurs couturière, ils prirent chacun un métier et devinrent très riches ", $\mathrm{n}^{\circ} 40$. Sur la question de l'opération d'actualisation des contes, voir Micheu Chapduelh, La Fada multicarta, 20113).

Par-delà les destins princiers des humbles protagonistes des contes, qui ne sont pas là pour qu'on les croit possibles (du reste, à l'époque du collectage, en régime républicain, ce sont depuis longtemps des éléments vidés de tout référent dans la société réelle), on ne peut espérer mieux que de devenir facteur et couturière. 
7 Comme le remarque J. Bru, le trait le plus saillant du recueil est d'ailleurs l'impression de grand dénuement et de pauvreté véhiculé par tous ces contes quel que soit leur genre (initiatiques, animaliers, facétieux, etc.). Le charbonnier est «paure, paure coma un rat » (« pauvre, pauvre comme un rat », $\mathrm{n}^{\circ}$ 79). La maîtresse sermonne son valet qui vient chercher pour son déjeuner " una pola còita »: «Creses que sèm plan riches per hèr còser polas cada jorn» ( $\mathrm{Tu}$ crois que nous sommes assez riches pour faire cuire des poules chaque jour?" Lo curè còit, $\left.\mathrm{n}^{\circ} 114\right)$. Un conte (La mairastra, $\mathrm{n}^{\circ} 16$ ), après l'évocation finale du mariage de la fille maltraitée avec un prince, s'achève par cette formule révélatrice du conteur : «E jo èri de nòças damés esclòps, e me volegan pas» (« $\mathrm{Et}$ moi j'étais de noce, en sabots, et ils ne me voulurent pas »). La fille délaissée, sans faire de manières, utilise la formule: "jo que sèu tota pesolhs» («moi qui suis toute pouilleuse " ( ${ }^{\circ} 20$ L'auquièra, p. 80). Lorsque Cendrilhon prononce les mêmes mots, il faut les prendre à la lettre, car en disant cela, "se tirava un grun de sava de peu cap e la getava au hòc e petilhava coma de pesolhs " (" elle se tirait un grain de sève de la tête et le jetait au feu et ça pétillait comme des poux ", $\mathrm{n}^{\circ} 28$ ). L'épouillage d'un enfant, comme dans le célébrissime poème de Rimbaud Les Chercheuses de poux (1871), est d'ailleurs perçu comme un geste de tendresse, dans une très belle phrase du conte des Tres rius (Les trois ruisseaux, $\mathrm{n}^{\circ}$ 5) : « La Santa Vièrja, plan contenta, le hasquèc cochar sus sos ginolhs, le penchenèc, le despesolhèc, juscas autant que s'endromic » (la Sainte Vierge, bien contente, le fit coucher sur ses genoux, le peigna, l'épouilla, jusqu'à ce qu'il s'endorme »).

De très nombreux contes démarrent par l'événement traumatique d'une extrême misère contraignant les protagonistes à " partir a l'aumòina " (" partir mendier »). Cette obsession d'être poussé sur les chemins par la famine n'est certes pas seulement un lieu commun du conte ou une terreur fantasmatique de la société paysanne sédentaire ${ }^{4}$; elle est une menace bien présente et surtout une mémoire historique qui remonte au moins aux famines de l'Ancien régime; il n'est guère risqué de l'avancer, car les informateurs les plus âgés de ces enfants étaient nés vers les années 1820-1830 et les grands-parents de ces gens-là avaient pu connaître les famines de la fin du XVIII e siècle qui jetaient encore les familles entières sur les routes. Il ne faut pas non plus oublier que 1812, 1817, 1829-1832, 1846-1847 furent encore des années de disette.

Cette terreur est toujours active et, de toute façon, la grande pauvreté est partout : les contes qui personnifient la misère, comme celui du forgeron éponyme qui, refusé au paradis et chassé de l'enfer « se'n tornèc sur la tèrra » ( « revint sur terre », nº 8 ), le disent sans ambages : "estec e es encara Misèra, e totjorn ne sirà » (" Misère a existé et existe encore, et il existera toujours ", $n^{\circ} 4$ ).

10 Ce dénuement se fait entendre d'ailleurs dans la langue même, par son immédiateté, sa crudité, sa brutalité même parfois, qui peuvent paraître choquantes aujourd'hui, quand on sait que ces histoires furent contées par des adultes à des enfants. Ceux-ci ne sont certes pas ménagés, surtout lorsqu'il s'agit de leur inculquer la méfiance et l'obéissance: "Les Diables se mingègan aquera dròlla $e$ i pengègan las tripas au carmalhèr» («Les démons mangèrent cette fille et pendirent ses tripes à la crémaillère ", $\mathrm{n}^{\circ} 12$ - La duas hilhas que van au castèth deu Diables). Cela est aussi vrai des histoires scatologiques et même carrément sexuelles qui leur sont transmises. Certes, le collectage des enfants, comme le fait remarquer J.Bru, ne saurait être exhaustif; certains contes ne peuvent être transmis qu'à des adultes de même sexe. Mais enfin, ajoute-t-elle, « on trouve rarement une telle crudité dans les recueils imprimés avant la moitié du xx siècle » (p. 14). Il passe en effet bien des expressions et des termes pour le 
moins directs, parfois même sont-ils mis dans la bouche de personnages enfantins: «Vietdase!»; «me'n fiqui plan»; "m'as colhonat »; «Minja mèrda qui m'escota!», etc. D'ailleurs, Perbosc n'a pas fait traduire certains textes à ses élèves, et souvent les gros mots sont suppléés par des points de suspension. Grain de Millet n'hésite pas à s'écrier : «vielha salòpa, me pishas suu cap!» (« vieille salope, tu me pisses sur la tête », $\mathrm{n}^{\circ} 36$ ). Ou soit encore cet échange courtois entre un benêt et une princesse: "Sètz plan polida, vietdase! Volètz que vous embraci? - Una mèrda, respondec la hilha deu rei » (littéralement : "Vous êtes bien jolie, vindieu! Vous voulez que je vous embrasse? - Une merde, répondit la fille du roi ", $\mathrm{n}^{\circ} 42$ ).

11 La scatologie est omniprésente, avec la transsubstantiation récurrente de l'excrément en or: le cheval de La Gata blanca $\left(\mathrm{n}^{\circ} 9\right)$ "en i passant la man sur l'esquina, cagava Lovidors" (" en lui passant la main sur l'échine, il chiait des Louis d'or »), comme l'âne dans Nòste-Senher e le paure, "se metec a cagar lovidòrs" ( $\left.\mathrm{n}^{\circ} 14\right)$. "Intra dins mon cuòu, te portarè » («Entre dans mon cul et je te porterai »), tel est le refrain de la Borseta panada, idem dans Mitat-Capon: "Le Renard $i$ horra le nas au cuòu, e s'i damorèc "; ( Le renard y fourra le nez au cul, et il y resta ", $\mathrm{n}^{\circ} 65$ et 80). Dans Le Lop, la Craba e les crabotets (Le Loup, la chèvre et les chevreaux, $\mathrm{n}^{\circ} 60$ ), la chèvre ordonne au loup : "Caga-me mos crabotets! Caga-me mos crabotets! E les i caguèc » ("Chie-moi mes chevreaux! chie-moi mes chevreaux ! Et il les lui chia »). Grain de Millet et le loup qui l'a avalé se répondent en écho : «Lop, caga-me! - Cara-te! - Caga-me! - Cara-te! Enfin, le caguèc » (« loup, chiemoi ! - Tais-toi ! - Chie-moi ! - Tais-toi ! Enfin, il le chia ", $\left.n^{\circ} 37\right)$. On notera encore les péripéties surréalistes qui terminent le conte intitulé Les mic et les mac $\left(n^{\circ} 17\right)$ : «La filha avè la camisa entà cuòu traucada e un jardinèr ende reparar i fotec una felha de caulet au cuòu. Passègan peu fierau deu bestiar e un buòu se volec minjar quera felha au cuòu de la filha » ("La fille avait la chemise trouée jusqu'au cul et un jardinier pour remédier y foutit une feuille de choux au cul. Ils passèrent par le foirail à bestiaux et un bœuf voulut manger cette feuille au cul de la fille »). On hésite, mais à la fin on ne résiste pas à citer l'épisode du conte drolatique Filon refilon, où le valet rusé et facétieux, sorte de trickster occitan, place une chandelle allumée dans le cul d'une vieille endormie : « la candèla se hondè e le hòc començava a s'atrapar a las estopas " (" la chandelle se fondit et le feu commença à se prendre à l'étoupe »)...

12 Les allusions et équivoques sont parfois carrément sexuelles, ce qui montre assez, me semble-t-il, que les informateurs savaient que le recueil était destiné à des adultes, pardessus les épaules des enfants, qui n'en perdirent cependant pas une goutte, conformément aux instructions qu'ils avaient reçues! Joan lo nèçi (Jean le sot, $\mathrm{n}^{\circ} 49$ ) déclare, sans se rendre compte de ce qu'il dit : «çò de davant per las joenas, çò de darrèr per las vièlhas » ( le devant pour les jeunes, le derrière pour les vieilles »). Et l'on trouve quelques belles équivoques, comme celle de Mari Berlengon qui dit à Jan Latjon qui veut lui mettre l'anneau au doigt: "Me le soi mes plan sovent sans tu; me le metrè ben ara!» ( "Je me le suis mis bien souvent sans toi, et je me mettrai bien encore maintenant ", $\left.\mathrm{n}^{\circ} 115\right)$; ou celle du merle caché sous le corsage d'une paroissienne en pleine messe $\left(\mathrm{n}^{\circ} 110\right)$, qui fait dire au curé : «Las qu'avètz un merle, sortètz». Toutes sortent alors, sauf une vieille qui dit: "O! Le meu, $i$ a plan 'n pauc que canta pas mèi " ( Oh le mien, il y a bien longtemps qu'il ne chante plus»). Une partie de ce matériel sera d'ailleurs intégré plus tard par Perbosc dans ses Contributions au folklore érotique (Contes licencieux de l'Aquitaine, [1907] 1984 et L'anneau magique. Nouveaux contes d'Aquitaine, 1987). 

l'anticléricalisme, voire l'irréligion. Le curé est particulièrement visé (« colhon de curè », $\left.\mathrm{n}^{0} 25\right)$; « lo rector » est régulièrement dupé, volé, maltraité - on va même jusqu'à le faire cuire! (Le curè còit, $\mathrm{n}^{\circ}$ 118) -, sans que personne, apparemment, ne s'en émeuve... Filon Refilon $\left(n^{\circ} 45\right)$ fait croire à son curé, qu'il est le bon Dieu venu le chercher pour l'emmener dans l'au-delà ; il le fourre dans un sac et en cet état le traîne par l'escalier du clocher («qu'es maishant, le camin deu paradis!»: «il est mauvais, le chemin du paradis»), un long et pénible "espercatòri », un bien beau mot-valise pour désigner le purgatoire (sur la base du verbe «esperar » : attendre). Un conte propose une stratégie ébouriffante pour se débarrasser d'un curé «plan bestia» (bien idiot, $\mathrm{n}^{\circ} 113$ ), en présence de son évêque en visite pastorale : les paroissiens blasphèment (renegan) en se signant et en baisant le Christ à grands coups de « Coquin de Dius »; « Mila Dius », « Triple Dius »... On signalera aussi l'histoire de Sent Cramari ( $\left.n^{\circ} 82\right)$, identifié à sa statue ; pour expliquer sa mutilation involontaire, le curé dit que «s'a copat un braç en se batent damé les autes sants!» («il s'est coupé le bras en se battant avec d'autres saints!»). À noter, dans le même conte, le mot de "pocessiu», pour procession; lapsus quelque peu inquiétant. toujours prêt à supposer chez le bon peuple des campagnes la « foi du charbonnier ». Mais alors que faire des sarcasmes, des mots de reniement, des imprécations? Une phrase comme celle-ci n'aurait aucune importance, aucune profondeur critique : «Cau plan que Nòstre-Sénher nos vèlha mau, que nos bote dins una parelha misèra!» («Il faut bien que Notre Seigneur nous veuille du mal, pour nous jeter dans une pareille misère ", $\mathrm{n}^{\circ} 2$, Les secrets de las bestias) ? Et comment évaluer l'histoire de Jean de l'Ours ( $\left.\mathrm{n}^{\circ} 9\right)$, qui se termine par ces mots au demeurant bien peu chrétiens : «se n'angèc au castèth damé las dròllas deu Diables, e achí vivegan plan contents totes ensemble » (« il s'en fut au château avec les filles des Diables, et là ils vivaient bien contents tous ensemble ») ?

15 La politique des contes n'est jamais facile à évaluer ; le constat social le plus rude s'y accompagne de perspectives de réussite mirifiques (Cendrillon), certes improbables et donc peu consolatoires, ou plus raisonnables (le petit poucet facteur et sa sœur couturière). Il n'est en tout cas pas évident que les hiérarchies sociales y soient appréhendées comme l'ordre naturel des choses. En témoigne par exemple le traitement de la très ancienne métaphore organiciste des membres et de l'estomac d'Ésope, de Tite-Live et de La Fontaine, toujours mobilisée pour justifier les inégalités et les relations de pouvoir établies. Or la morale qui en tirée, à Comberouger $\left(n^{\circ} 88\right)$, est entièrement différente : «La societat des membres e de l'estomac es coma la societat umana. Un òme pòt pas hèr sans sos amics. Es pas una granda richessa ni una granda plaça que para l'òme: es l'amistat » («La société des membres et de l'estomac est comme la société humaine. Un homme ne peut pas faire sans ses amis. Ce n'est pas une grande richesse ni une haute fonction qui met l'homme à l'abri : c'est l'amitié »).

Les références classiques qu'impose l'évocation de l'apologue des membres et de l'estomac me conduisent à une dernière remarque. Aussi ancrée que soit cette culture dans l'oralité, bien des fois, l'écrit, la littérature mémorisée affleure ; une seule fois, des phrases françaises directement dérivées de Perrault se font entendre dans une version du chat botté $\left(\mathrm{n}^{\circ} 19\right)$, mais à plusieurs reprises les contes résonnent avec Perrault et Grimm (par exemple, avec Barbe-bleue, $\mathrm{n}^{\circ} 23$, Cendrillon, $\mathrm{n}^{\circ} 27$ et 28, ou les Musiciens de Brème, $\left.\mathrm{n}^{\circ} 70\right) \ldots$ Et lorsque le conte rencontre un fait divers macabre, on peut supposer 
une lecture initiale d'un canard sanglant (Le Tuaire de hemnas: le tueur de femmes, $\mathrm{n}^{\circ}$ 93, qui se termine par l'intervention des « gendarmas » et l'arrestation du criminel).

J'espère avoir donné un aperçu de la richesse de ce recueil, impeccablement présenté par J. Bru et J. Eygun, l'indispensable éditeur de Letras d'òc. Ce livre est précieux, sans nul doute, pour les amateurs de littérature orale, mais aussi pour ceux qui pratiquent l'histoire sociale et l'anthropologie, sans parler du linguiste qui peut y trouver bien des choses sur ce parler de frontière. Il présente cependant, à mon avis, une anomalie, que j'ai tenté déjà de pointer ailleurs ${ }^{5}$; nous avons ici un texte entièrement en occitan et non traduit, c'est-à-dire qui ne peut être lu sans connaître la langue. Or la préface est en français, alors que, pourtant, la note sur l'établissement du texte est en occitan. Pourquoi faut-il qu'un livre, même s'adressant à des occitanophones, contienne presque nécessairement du français dans ses parties critiques, surtout lorsque celles-ci apportent des éléments d'interprétation ou de théorie? Il est important de montrer, selon moi, que tout cela peut se faire aussi en occitan. On ne peut dire non plus que tout lecteur de l'occitan est francophone ; il existe un public pour l'occitan - aussi restreint soit-il - italianophone, castillanophone et surtout catalanophone, qui ne connaît pas ou mal le français. Une autre option, qui aurait aussi rendu le livre plus cohérent, aurait consisté à intégrer à l'ouvrage les traductions françaises, il est vrai de qualité inégale (ce sont des devoirs d'enfant, d'ailleurs intéressants à ce titre), des contes qui figurent dans le recueil; dans ce cas, la langue de l'appareil critique serait mieux justifiée, quoique, là encore, pour ma part, je trouve à redire, car cela revient à reproduire, sans l'interroger ni l'inquiéter, l'ordre diglossique que ces contes justement inquiètent, dans leur capacité à s'approprier les modèles nobles de la littérature française.

\section{NOTES}

1. Josiane Bru, "Perbosc et l'enquête folklorique ", Cahiers de littérature orale, $\mathrm{n}^{\circ} 63-64,2008$, consulté le 23 mars 2015.

2. Cela n'est pas sans importance, car l'internaute qui cherche du matériel occitan est écarté du document.

3. Voir notre compte rendu sur notre blog Mescladis e còps de gula, consulté le 23 mars 2015.

4. Voir, par comparaison, les versions manouches des mêmes contes ; la mendicité n'y est pas du tout appréhendée comme cet événement traumatique et cette plongée dans la déréliction. $\mathrm{Cf}$. Contes des manouches d'Auvergne (bilingues) par Joseph Valet.

5. «Cossi editar los classics occitans? », consulté le 23 mars 2015. 